\title{
Self-imaging of gratings with rough strips
}

\author{
Francisco Jose Torcal-Milla, ${ }^{*}$ Luis Miguel Sanchez-Brea, and Eusebio Bernabeu \\ Optics Department, Applied Optics Complutense Group, Universidad Complutense de Madrid, \\ Facultad de Ciencias Físicas, Ciudad Universitaria s.n., 28040 Madrid, Spain \\ *Corresponding author: ftorcalmilla@fis.ucm.es
}

Received May 14, 2008; accepted July 20, 2008;

posted July 25, 2008 (Doc. ID 96107); published September 3, 2008

\begin{abstract}
We analyze the self-imaging process produced by a transmission grating whose strips present two different roughness levels. This kind of grating periodically modulates the transmitted light owing only to the different microtopographic properties of the strips. In spite of the fact that the grating is not purely periodic, it produces a kind of self-image at Talbot distances. These self-images gradually appear as light propagates, but they are not present just after the grating, as occurs in amplitude or phase gratings. There exists a distance from the grating, which depends on the stochastic properties of roughness, from which the contrast of the self-images becomes stable. Important cases are analyzed in detail, such as low- and high-roughness limits. We assume for the calculations that the grating can be used in a mobile system. Simulations using the Rayleigh-Sommerfeld regime have been performed, which confirm the validity of the theoretical approach proposed in this work (C) 2008 Optical Society of America

OCIS codes: $030.5770,050.0050,050.2770,290.5880$.
\end{abstract}

\section{INTRODUCTION}

When a diffraction grating is illuminated with a monochromatic plane wave, self-images of the grating are formed at certain distances after it. This effect, known as the Talbot effect and discovered by W. F. H. Talbot in 1836 , is produced when the propagated light beam acquires a periodic modulation of some of its properties [1-3]. Normally amplitude or phase gratings are used, which modulate the amplitude or the phase of the incident light, respectively [4-7]. There are also other possibilities for modulating the incident light, such as polarization gratings, which modulate the state of polarization [8-10], and gratings whose strips present a different microtopographic structure [11]. Examples of gratings with roughness are steel tape gratings used in displacement measurement systems [12]. In a previous paper we analyzed the far-field diffraction pattern of transmission gratings whose strips present two different roughness levels, one of them smooth and the other one rough [13]. The efficiency of the diffraction orders was shown to depend on the statistical properties of the roughness.

Another important aspect of diffraction gratings is the Talbot effect. Self-images are obtained in the near field at periodic distances known as Talbot planes [3]. When the grating presents a certain roughness, a statistical approach for determining the intensity distribution after the grating is required. The Talbot effect for steel tape gratings has been analyzed previously, showing that the contrast of the self-images decreases when the distance between the grating and the observation plane increases $[11,12]$. In the present work, the gratings are assumed to be transmission gratings. Since the grating presents a rough surface, we determine the mutual intensity function in the Fresnel regime using the Fresnel approach to study the self-imaging process. We see that the behavior of the self-images for transmission through rough grat- ings is completely different from that for metallic gratings. Self-images are not present close to the diffraction grating but gradually appear as light propagates. From a given threshold distance between the grating and the observation plane, self-images become stable and present a constant contrast. Two important cases, low- and highroughness limits, will be analyzed in detail. Finally, the validity of this approach is checked by means of numerical simulations based on the Rayleigh-Sommerfeld approach to diffraction. To perform the numerical implementation, we have used a fast-Fourier-transform-based direct integration method [14]. Since the results presented are stochastic, we have computed the intensity distribution for an ensemble of realizations. The final result is the average of all the realizations. The results obtained with the simulations are in complete agreement with the presented theory.

\section{THEORETICAL APPROACH}

Let us consider a grating formed by strips with two different roughness levels, one with a constant height $\zeta_{0}$ that, without loss of generality we assume zero, $\zeta_{0}=0$, and the other one with a rough surface that presents a random topography given by $\zeta(x)$, whose average height is null $\langle\zeta(x)\rangle=0$. The transmittance of the rough level is $t(x)=\exp [i k(n-1) \zeta(x)]$, where $k=2 \pi / \lambda$ and $n$ is the refractive index of glass [15]. Mathematically, this grating can be described as a sum of two amplitude binary gratings with period $p$ (average amplitude levels 0 and 1). The first grating is

$$
G_{1}(x)=\sum_{l} a_{l} \exp (i q l x)
$$

where $q=2 \pi / p$. The Fourier coefficients of the grating $G_{1}(x)$ are $a_{l}=\kappa \operatorname{sinc}(l \pi \kappa)$, with $\kappa=\alpha / p$, as defined in Fig. 1. 


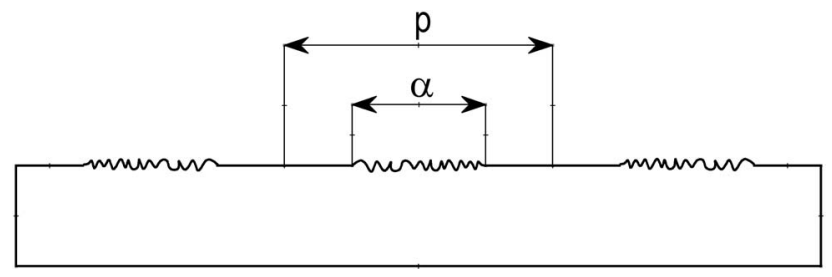

Fig. 1. Diffraction grating proposed in this paper. The strips present different microtopographic structures.

These strips present a rough topography; thus its transmittance results in $G(x)=t(x) G_{1}(x)$. The second amplitude grating, $G_{2}(x)=1-G_{1}(x)$, is formed by strips with two constant levels, in the same way as $G_{1}(x)$. The sum of these two amplitude gratings [13],

$$
T(x)=1-G_{1}(x)[1-t(x)],
$$

describes the whole structure, which acts stochastically on the phase of the incident wave. When roughness is null, then $t(x)=1$ and the grating disappears, since the transmittance is $T(x)=1$.

For the general case, the transmission coefficient $T(x)$ produces a random change in the phase of the incident beam when it passes through the rough strips. For simplicity and without loss of generality, we consider the onedimensional problem. In addition, the transmission variation owed to Fresnel coefficients is not included.

To describe the random topography of the rough strips a normal distribution in heights is assumed, $w(z)$ $=\exp \left(-z^{2} / 2 \sigma^{2}\right) / \sqrt{2 \pi} \sigma$, where $z=\zeta(x)$, and $\sigma$ is the standard deviation. The characteristic function that describes the average transmittance of this distribution results in

$$
\langle t(x)\rangle=\int w(z) \exp [i k(n-1) z] \mathrm{d} z=\exp (-g / 2),
$$

where $g=[k \sigma(n-1)]^{2}$. We also need the two-dimensional distribution of heights $w\left(\zeta(x), \zeta\left(x^{\prime}\right)\right)$ at two different points $z=\zeta(x), z^{\prime}=\zeta\left(x^{\prime}\right)$. Assuming a Gaussian distribution with variance $\sigma^{2}$ and mean value zero,

$$
\begin{aligned}
w\left(\zeta(x), \zeta\left(x^{\prime}\right)\right)= & \frac{1}{2 \pi \sigma \sqrt{1-C(\tau)^{2}}} \\
& \times \exp \left[-\frac{\zeta^{2}(x)-2 C(\tau) \zeta(x) \zeta\left(x^{\prime}\right)+\zeta^{2}\left(x^{\prime}\right)}{2 \sigma^{2}\left(1-C(\tau)^{2}\right)}\right],
\end{aligned}
$$

where we also assume that the autocorrelation coefficient is Gaussian, $C(\tau)=\left\langle\zeta(x) \zeta\left(x^{\prime}\right)\right\rangle /\left\langle\zeta^{2}(x)\right\rangle=\exp \left(-\tau^{2} / T_{0}^{2}\right), \quad \tau=x$ $-x^{\prime}$, and $T_{0}$ the correlation length. As a result, the characteristic function of this distribution is [16]

$$
\begin{aligned}
\left\langle t(x) t^{*}\left(x^{\prime}\right)\right\rangle & =\exp \{-g[1-C(\tau)]\} \\
& =\exp (-g) \sum_{m=0}^{\infty} \frac{g^{m}}{m !} \exp \left[-m \frac{\tau^{2}}{T_{0}^{2}}\right] .
\end{aligned}
$$

Considering plane wave illumination $U_{0}(x)=A_{0}$, the field just after the grating is $U_{1}(x)=A_{0} T(x)$. Since the topography is stochastic, a more useful function is the mutual intensity function, which is defined as $J\left(x, x^{\prime}\right)$ $=\left\langle U_{1}(x) U_{1}^{*}\left(x^{\prime}\right)\right\rangle$. The normalized mutual intensity function is defined as $\overline{J\left(x, x^{\prime}\right)}=J\left(x, x^{\prime}\right) /\left|A_{0}\right|^{2}=\left\langle T(x) T *\left(x^{\prime}\right)\right\rangle$. Considering that the only stochastic process is produced by the rough topography and using Eq. (2), the normalized mutual intensity function can be rewritten as

$$
\begin{aligned}
\overline{J\left(x, x^{\prime}\right)}= & 1+G_{1}(x)[\langle t(x)\rangle-1]+G_{1}^{*}\left(x^{\prime}\right)\left[\left\langle t^{*}\left(x^{\prime}\right)\right\rangle-1\right] \\
& +G_{1}(x) G_{1}^{*}\left(x^{\prime}\right)\left[1-\langle t(x)\rangle-\left\langle t^{*}\left(x^{\prime}\right)\right\rangle+\left\langle t(x) t^{*}\left(x^{\prime}\right)\right\rangle\right],
\end{aligned}
$$

and substituting Eqs. (3) and (5) into Eq. (6), it simplifies to

$$
\begin{aligned}
\overline{J\left(x, x^{\prime}\right)}= & 1-\left(1-e^{-g / 2}\right)\left[G_{1}(x)+G_{1}^{*}\left(x^{\prime}\right)\right]+G_{1}(x) G_{1}^{*}\left(x^{\prime}\right) \\
& \times\left[\left(1-2 e^{-g / 2}\right)+e^{-g} \sum_{m=0}^{\infty} \frac{g^{m}}{m !} \exp \left(-m \tau^{2} / T_{0}^{2}\right)\right] .
\end{aligned}
$$

To determine the near-field diffraction pattern, we consider the Fresnel regime. The normalized mutual intensity at a distance $z$ from the grating is [17]

$$
\begin{aligned}
\overline{J\left(x_{2}, x^{\prime}{ }_{2}, z\right)=} & \frac{1}{\lambda z} \int \overline{J\left(x_{1}, x^{\prime}{ }_{1}\right)} \exp \left[\frac{i k}{2 z}\left(x^{\prime}{ }_{2}-x^{\prime}{ }_{1}\right)^{2}\right] \\
& \times \exp \left[-\frac{i k}{2 z}\left(x_{2}-x_{1}\right)^{2}\right] \mathrm{d} x_{1} \mathrm{~d} x^{\prime}{ }_{1} \\
= & {\left[1-\left(1-\mathrm{e}^{-g / 2}\right) H\left(x_{2}, z\right)\right]\left[1-\left(1-\mathrm{e}^{-g / 2}\right) H^{*}\left(x^{\prime}{ }_{2}, z\right)\right] } \\
& +e^{-g} \sum_{m=1}^{\infty} \frac{g^{m}}{m !} \sum_{l} \sum_{l^{\prime}} a_{l} a_{l^{\prime}} \\
& \times \exp \left[i q\left(l x_{2}-l^{\prime} x^{\prime}{ }_{2}\right)\right] \exp \left[i\left(l^{2}-l^{\prime 2}\right) \frac{z}{z_{T}}\right] \\
& \times \exp \left[\frac{-m\left[\left(l-l^{\prime}\right) q z-k\left(x_{2}-x^{\prime}{ }_{2}\right)\right]^{2}}{\left(k T_{0}\right)^{2}}\right],
\end{aligned}
$$

where $z_{T}=p^{2} / \lambda$ is the Talbot distance and $H\left(x_{2}, z\right)$ $=\Sigma_{l} a_{l} \exp \left(-i \pi l^{2} z / z_{T}\right) \exp \left(i l q x_{2}\right)$ is the amplitude produced by $G_{1}(x)$ at the observation plane. The normalized average intensity at a distance $z$ is easily obtained from the normalized mutual intensity, $\overline{\left\langle I\left(x_{2}, z\right)\right\rangle}=\overline{J\left(x_{2}, x_{2}, z\right)}$, which results in

$$
\begin{aligned}
\overline{\left\langle I\left(x_{2}, z\right)\right\rangle}= & \left|1-\left(1-e^{-g / 2}\right) H\left(x_{2}, z\right)\right|^{2}+e^{-g} \sum_{m=1}^{\infty} \frac{g^{m}}{m !} \sum_{l, l^{\prime}} a_{l} a_{l^{\prime}}^{*} \\
& \times \exp \left[i\left(l-l^{\prime}\right) q x_{2}\right] \exp \left[-i \pi\left(l^{2}-l^{\prime 2}\right) \frac{z}{z_{T}}\right] \\
& \times \exp \left[-m\left(l-l^{\prime}\right)^{2}\left(\frac{z}{z_{C}}\right)^{2}\right],
\end{aligned}
$$

where $z_{C}=p T_{0} / \lambda$. The averaging in the intensity is performed on an ensemble of realizations that are obtained, for example, when the grating is moved in the direction parallel to the $y$ axis. To understand the behavior of this kind of grating, an example of the intensity obtained with Eq. (9) is shown in Fig. 2(a). Self-images are not present 
(a)

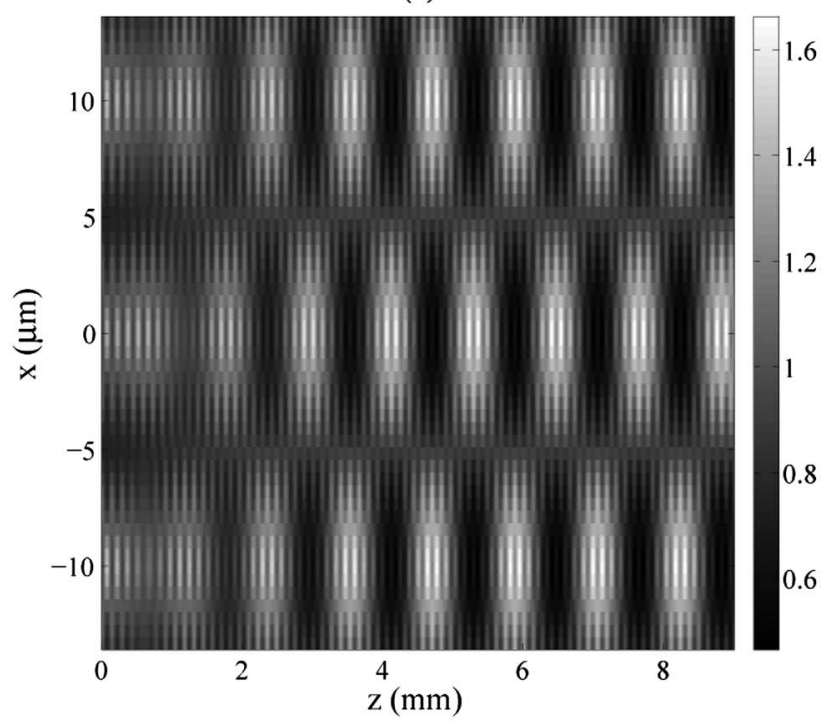

(b)

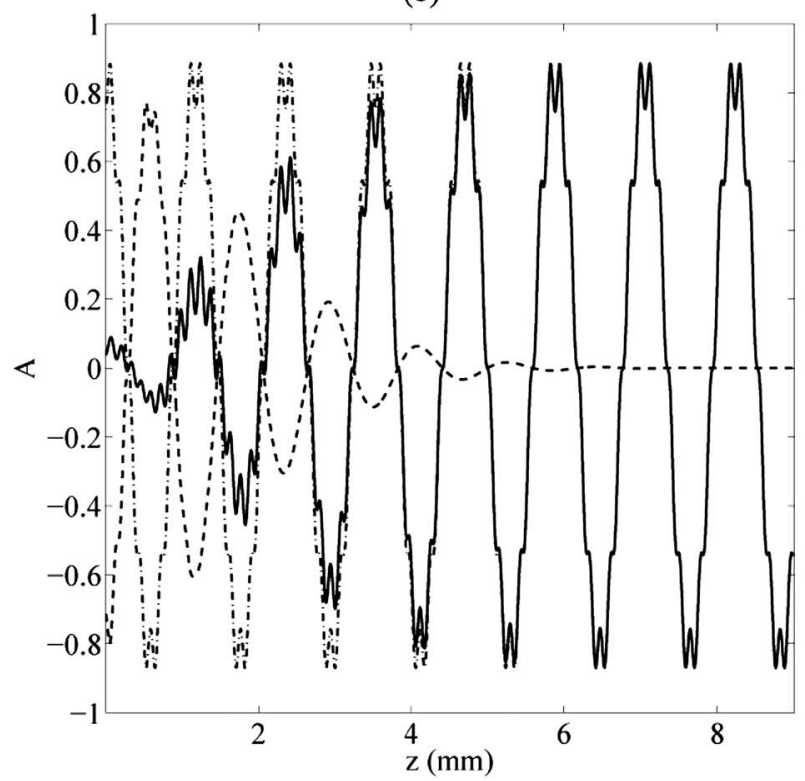

Fig. 2. (a) Near-field intensity pattern using Eq. (9) for a grating with period $p=20 \mu \mathrm{m}$ when $\sigma=0.25 \mu \mathrm{m}, T_{0}=100 \mu \mathrm{m}$, and $n$ $=1.5$. The wavelength is $\lambda=0.68 \mu \mathrm{m}$. (b) Amplitude of the selfimages (solid curve) and terms of Eq. (9): first term (dashed-dot curve), second term (dashed curve).

just after the grating but gradually appear as the light propagates. Since the grating produces a modulation only in the phase of the incident beam, the intensity just after the grating is the same than just before the grating. In Fig. 2(b), the two terms of the amplitude of Eq. (9) and the total amplitude of this intensity distribution are represented. The amplitude is defined as $A(z)=I_{M}(z)-I_{m}(z)$, where $I_{M}(z)$ is the intensity at the center of the smooth slits and $I_{m}(z)$ is the intensity at the center of the rough slits. The contrast definition used in all the cases is $C(z)$ $=\left[I_{M}(z)-I_{m}(z)\right] /\left[I_{M}(z)+I_{m}(z)\right]$. We assumed this contrast definition to bring out the inversion of the contrast in the self-imaging process. We see that the two terms of Eq. (9) cancel each other just after the grating. The first term of Eq. (9) is the intensity after an amplitude grating defined as $G^{\prime}(x)=1-\left(1-e^{-g / 2}\right) G_{1}(x)$. It is produced by an interferential effect among the grating slits. The second term is produced by a scattering process of the rough slits that makes the contrast of the self-images decrease. However, the second term decreases exponentially. The distance $z_{C}=p T_{0} / \lambda$ is approximately the distance at which selfimages appear. When $z \gg z_{C}$ the last factor of Eq. (9) disappears except for $l=l^{\prime}$. Then, for this limit case the average intensity results in

$$
\overline{\left\langle I\left(x_{2}, z\right)\right\rangle}=\left|1-\left(1-e^{-g / 2}\right) H\left(x_{2}, z\right)\right|^{2}+\left(1-e^{-g}\right) \kappa .
$$

The effect of roughness in this case is just to produce the self-imaging process as if it were an amplitude grating with modified Fourier coefficients

\section{A. Slight-Roughness Regime}

Figure 3 presents the self-imaging process for several values of $\sigma$ that cover the most important situations that we can find. The general case has been explained in the previous section. Two important cases are those for slight and high roughness, which will be analyzed here in detail. Roughness is slight when $\sigma \ll \lambda$ and as a consequence $g$ $\ll 1$. Performing a linear series expansion in $g$, the mean intensity distribution in the near field results in

$$
\begin{aligned}
\overline{\left\langle I\left(x_{2}, z\right)\right\rangle}= & 1-g \sum_{l} a_{l} \cos \left(l q x_{2}+l^{2} \frac{z}{z_{T}}\right) \\
& +g \sum_{l, l^{\prime}} a_{l} a_{l^{\prime}}^{*} \exp \left[i\left(l-l^{\prime}\right) q x_{2}\right] \exp \left[-\left(l-l^{\prime}\right)^{2}\left(\frac{z}{z_{C}}\right)^{2}\right] \\
& \times \exp \left[-i\left(l^{2}-l^{\prime 2}\right) \frac{z}{z_{T}}\right] .
\end{aligned}
$$

As can be observed, the cosinusoidal term remains along the $z$ axis. The Talbot effect is produced, but the intensity is modulated by a multiplicative factor $g$. On the other hand, the third term decays with $z$. The contrast of the self-images decreases with $g$. An example of the contrast produced considering the slight-roughness regime is shown in Figure 3(a). Obviously, when roughness is null, $g=0$, the intensity distribution, $\left\langle I\left(x_{2}, z\right)\right\rangle=\left|A_{0}\right|^{2}$, shows that there exists no diffraction grating.

\section{B. High-Roughness Regime}

When roughness is high, $g \gg 1$, then the autocorrelation function given in Eq. (5) is approximately $\left\langle t(x) t^{*}\left(x^{\prime}\right)\right\rangle$ $=\exp \left[-\left(x-x^{\prime}\right)^{2} / T_{F}^{2}\right]$, where $T_{F}=T_{0} / \sqrt{g}=T_{0} /[k \sigma(n-1)]$. Then, the average intensity distribution in the near field results in

$$
\overline{\left\langle I\left(x_{2}, z\right)\right\rangle}=\left|1-H\left(x_{2}, z\right)\right|^{2}+\kappa .
$$

An example of the contrast produced considering this regime is shown in Fig. 3(c).

The first term is the near-field intensity distribution produced by a binary amplitude diffraction grating whose Fourier coefficients are those of the grating $G_{2}(x)=1$ $-G_{1}(x)$, which corresponds to the smooth slits of the grating. The second term is a constant-intensity factor, which is produced by the rough strips. This intensity distribution can be interpreted in the following way. Light that 
(a)

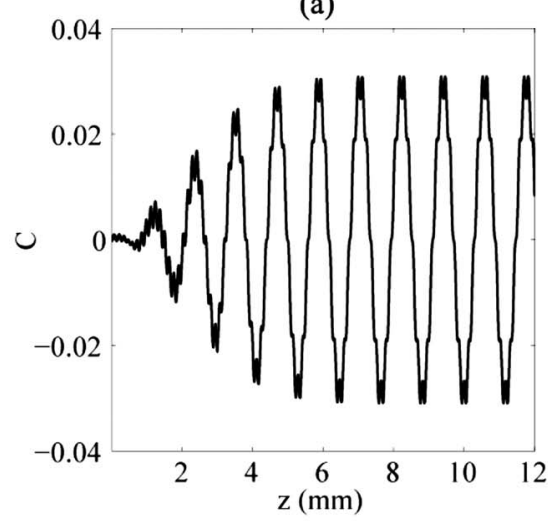

(b)

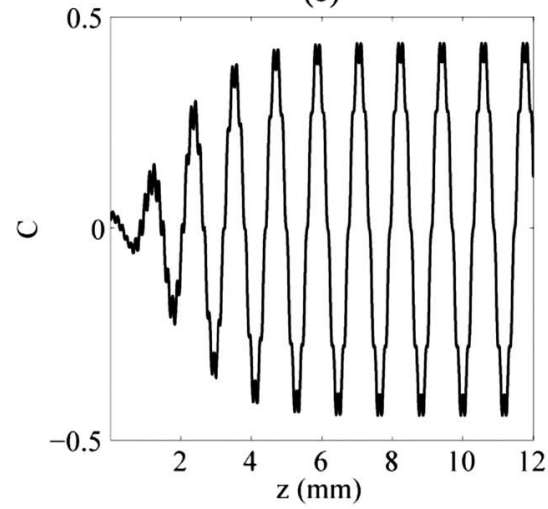

(c)

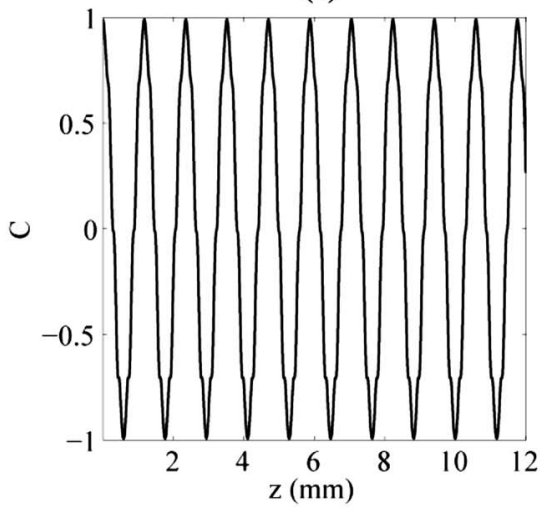

Fig. 3. Contrast of the self-images for a grating with parameters $p=20 \mu \mathrm{m}, n=1.5, l=l^{\prime}=3, T_{0}=100 \mu \mathrm{m}$, and wavelength $\lambda$ $=0.68 \mu \mathrm{m}$ for different values of $\sigma$. (a) $\sigma=0.05 \mu \mathrm{m}$, (b) $\sigma$ $=0.25 \mu \mathrm{m}$, (c) $\sigma=1 \mu \mathrm{m}$.

passes through the smooth strips interferes as if there were only an amplitude grating. Light that passes through the rough strips is scattered in all directions and contributes to the intensity as a background level.

\section{NUMERICAL SIMULATIONS}

To corroborate the results obtained with the theoretical formalism, we perform several numerical simulations based on the Rayleigh-Sommerfeld method for diffraction [4]. In the first place, we need to simulate the topography of the grating. For the rough strips, we have generated a stochastic function costructed by the superposition of Gaussian functions randomly spaced along the $x$ axis around a given separation between every two closed peaks. These Gaussian functions have a Gaussian distribution of heights and widths. An example of a grating is shown in Fig. 4(a).

To determine the near-field diffracted intensity distribution, we have numerically solved the RayleighSommerfeld diffraction integral using a fast-Fouriertransform-based direct integration (FFT-DI) method. The FFT-DI method is accurate and efficient and can be used for near-field computations [14].

Each simulation corresponds to a realization that can be assigned, for example, to the movement of the grating in the direction parallel to the $y$ axis, assuming that the grating can be used in a mobile device. Since the diffraction grating is not purely periodic, the intensity distribution of each realization after the grating presents a certain variability. As an example, in Fig. 4(b) we show the

(a)

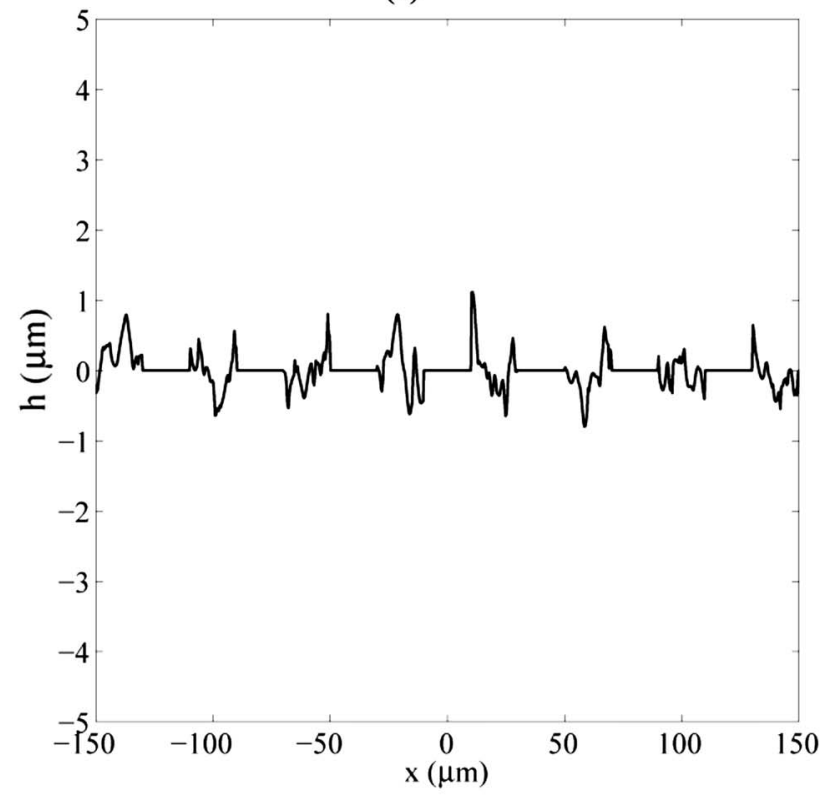

(b)

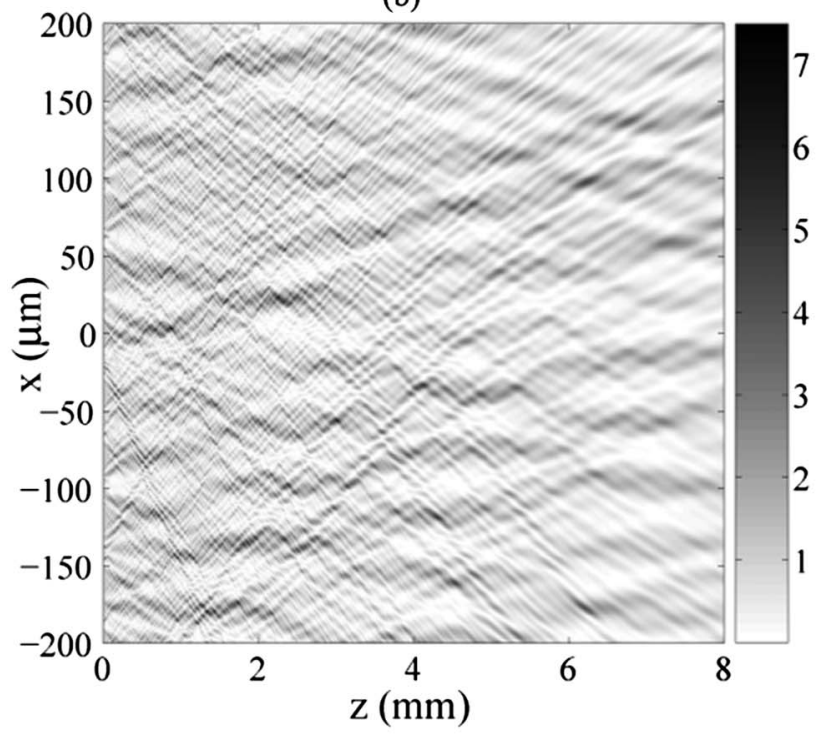

Fig. 4. (a) Example of a grating with period $p=40 \mu \mathrm{m}$, refractive index $n=1.5$, and roughness parameters $\sigma=0.25 \mu \mathrm{m}, T_{0}$ $=1 \mu \mathrm{m}$. (b) Near-field intensity pattern produced by this grating. 
intensity distribution at the near field for just one realization. The results obtained in Section 2 correspond to an averaging process. Next, we need to perform an ensemble of simulations in order to compare with the average intensity given in Eq. (9). We have performed numerous simulations, and an example of this averaging is shown in Fig. 5(a) for the case of 100 simulations when the dimensional parameters of the grating are $p=20 \mu \mathrm{m}$, $\sigma=0.25 \mu \mathrm{m}$, and $T_{0}=100 \mu \mathrm{m}$, and the wavelength is $\lambda=0.68 \mu \mathrm{m}$. This average result in Fig. 5(a) can be compared with the theoretical results of Fig. 2. In Fig. 5(b) the theoretical and numerical contrast are shown for the same values of $\sigma, T_{0} p$, and $\lambda$. In both cases the contrast of the self-images increases when the distance between the grating and the observation plane increases, the results being very similar.

(a)

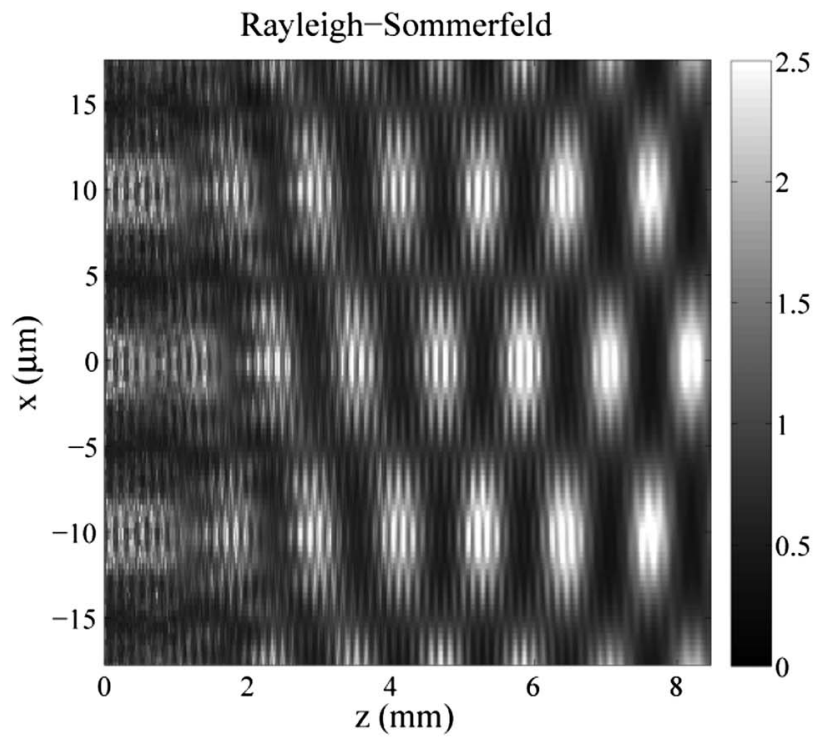

(b)

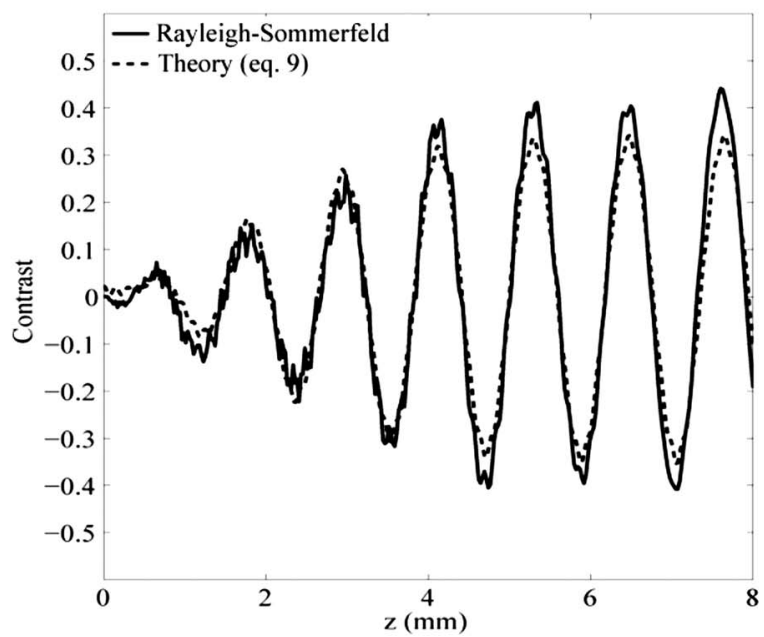

Fig. 5. (a) Intensity pattern obtained using the RayleighSommerfeld formalism taking the average of 100 simulations with $\sigma=0.25 \mu \mathrm{m}, T_{0}=100 \mu \mathrm{m}, p=20 \mu \mathrm{m}$. (b) Contrast comparison between numerical simulation and theoretical results.

\section{CONCLUSIONS}

A description of the near-field diffraction pattern of a grating formed by strips with two different roughness levels has been analyzed in this work. Since the topography of the grating is stochastic, a statistical approach based on the mutual intensity function is performed. Although the grating is not purely periodic, self-images in the near field are formed. The contrast of the self-images increases when the distance between the grating and the observation plane increases, and it stabilizes at a certain distance that depends on the roughness parameters. Slight- and high-roughness limits been have also analyzed. Numerical simulations based on the Rayleigh-Sommerfeld method for diffraction are performed, which corroborate the results obtained with the theoretical formalism.

\section{ACKNOWLEDGMENTS}

The authors thank Alfredo Luis and José María RicoGarcia for their fruitful ideas and discussions. This work has been supported by the DPI2005-02860 project of the Ministerio de Educación y Ciencia of Spain and Estratégicos Nacionales en Investigación Técnica (CENIT) project "Tecnologías avanzadas para los equipos y procesos de fabricación de 2015: e-eficiente, e-cológica, e-máquina (eEe)" of the Ministerio de Industria, Turismo y Comercio of Spain.

\section{REFERENCES}

1. W. H. F. Talbot, "Facts relating to optical science," Philos. Mag. 9, 401-407 (1836).

2. K. Patorski, "The self-imaging phenomenon and its applications," Prog. Opt. 27, 1-108 (1989).

3. E. Keren and O. Kafri, "Diffraction effects in moiré deflectometry," J. Opt. Soc. Am. A 2, 111-120 (1985).

4. M. Born and E. Wolf, Principles of Optics (Pergamon, 1980).

5. J. W. Goodman, Introduction to Fourier Optics (McGrawHill, 1968).

6. E. G. Loewen and E. Popov, Diffraction Gratings and Applications (Marcel Dekker, 1997).

7. C. Palmer, Diffraction Grating Handbook (Richardson Grating Laboratory, New York, 2000).

8. F. Gori, "Measuring Stokes parameters by means of a polarization grating," Opt. Lett. 24, 584-586 (1999).

9. C. G. Someda, "Far field of polarization gratings," Opt. Lett. 24, 1657-1659 (1999).

10. G. Piquero, R. Borghi, A. Mondello, and M. Santarsiero, "Far field of beams generated by quasi-homogeneous sources passing through polarization gratings," Opt. Commun. 195, 339-350 (2001).

11. F. J. Torcal-Milla, L. M. Sanchez-Brea, and E. Bernabeu, "Talbot effect with rough reflection gratings," Appl. Opt. 46, 3668-3673 (2007).

12. L. M. Sanchez-Brea, F. J. Torcal-Milla, and E. Bernabeu, "Talbot effect in metallic gratings under Gaussian illumination," Opt. Commun. 278, 23-27 (2007).

13. L. M. Sanchez-Brea, F. J. Torcal-Milla, and E. Bernabeu, "Far field of gratings with rough strips," J. Opt. Soc. Am. A 25, 828-833 (2008).

14. F. Shen and A. Wang, "Fast-Fourier-transform based numerical integration method for the Rayleigh-Sommerfeld diffraction formula," Appl. Opt. 45, 1102-1110 (2006).

15. B. E. A. Saleh and M. C. Teich, Fundamentals of Photonics (Wiley, 1991).

16. P. Beckmann and A. Spizzichino, The Scattering of Electromagnetic Waves from Rough Surfaces (Artech House, 1987).

17. J. W. Goodman, Statistical Optics (Wiley, 1985). 